\title{
Review of: "Pitfalls in comparison of coronary artery measurements of Indian population with different geographical area studies"
}

Shigenori Ito

Potential competing interests: The author(s) declared that no potential competing interests exist.

I would like to provide the opinion and limitations regarding this published article in the Indian heart journal.

1. I don't understand the significance of the primary aim of this study. The authors should have referred the data of acute and chronic clinical outcomes (i.e. initial success rate, patency at chronic stage, and complications) more in the PCI/CABG for the small/large coronary arteries.

2. As my personal thinking, I don't think there is a significant difference in the size of coronary arteries among the proposed geographical regions, which seems to be relatively narrow on the globe.

3. Body surface area is affected by body weight, which should change by aging (usually fat accumulation). Thus, I think "height" could be an alternative anthropometric variable to calculate indexed coronary artery diameter.

4. Reliable QCA analysis is mandatory when evaluating the study results. I think the following factors are lacking in this study.

5. The authors included the coronary arteries $\geqq 0.5 \mathrm{~mm}$. However, QCA should overestimate the arteries $\leqq 1.0 \mathrm{~mm}$ because of intrinsic limitations of current QCA algorithm. Small arteries should not be included.

6. Accuracy and precision of QCA depend on the catheter size as calibration device. The catheter size is not described in this study.

7. Dominancy of coronary arteries are not considered. For example small RCA in left dominancy and LCX of right dominancy should not be included for analysis. 